\title{
VIH EN GESTANTES INDÍGENAS: UN RETO PARA LA SALUD PÚBLICA PERUANA
}

\author{
Julio Portocarrero',a
}

\begin{abstract}
RESUMEN
Esta revisión narrativa aborda la problemática de las gestantes indígenas con $\mathrm{VIH}$, haciendo un recuento de los principales hallazgos sobre el tema a nivel internacional para luego revisar el estado de la investigación en el Perú. En el ámbito internacional se encuentra que el estudio sobre las gestantes indígenas con VIH se centra en la salud del nonato y la prevención de la transmisión vertical, existe poca información acerca de las concepciones sobre la enfermedad, prácticas de cuidado y prevención de la transmisión vertical, así como las percepciones sobre el tratamiento antirretroviral de gran actividad (TARGA) y el papel de la medicina tradicional en la atención de su condición de salud. A nivel nacional se encuentra solo con una referencia bibliográfica. Se evidencia la necesidad de un urgente enfoque médico antropológico para investigar y atender los casos de VIH en gestantes y mujeres en edad reproductiva en las comunidades indígenas de la amazonia peruana.
\end{abstract}

Palabras clave: VIH; Gestantes; Población indígena, Perú; Salud indígena (fuente: DeCS BIREME).

\section{HIV IN INDIGENOUS PREGNANT WOMEN: A CHALLENGE FOR PERUVIAN PUBLIC HEALTH}

\begin{abstract}
This narrative review addresses the issue of indigenous pregnant women with HIV, recounting the main findings on the issue at the international level and then reviewing the status of research in Peru. Research at the international level on indigenous pregnant women focuses on the health of the unborn and the prevention of vertical transmission. There is little information about their conceptions of the disease, care practices and prevention of vertical transmission, and their perceptions of Highly Active Antiretroviral Therapy (HAART) and the role of traditional medicine in the care of their health condition. At the national level only one bibliographic reference was found. There is evidence for the need of an urgent medical anthropological focus to investigate and deal with cases of HIV in pregnant women and women of reproductive age in indigenous communities in the Peruvian Amazon.
\end{abstract}

Key words: VIH; Pregnant women; Indigenous population, Peru; Health of indigenous peoples (source: MeSH NLM).

\section{GESTACIÓN, VIH Y MUERTE MATERNA EN LA POBLACIÓN INDÍGENA}

La mayor incidencia de muerte materna ocurre en países pobres, en vías de desarrollo, particularmente entre población indígena u originaria ${ }^{(1)}$. El reto de su reducción enfrenta actualmente la necesidad de atender los casos de gestantes viviendo con el virus de la inmunodeficiencia humana $(\mathrm{VIH})$ existentes en estos grupos poblacionales, ya que se estima que uno de los factores determinantes para el incremento de la muerte materna durante la última década es esta enfermedad (2).
La maternidad en mujeres viviendo con VIH es más riesgosa y puede convertirse en una causa importante de muerte materna, siendo un factor importante en África subsahariana ${ }^{(3)}$ y en el resto del mundo ${ }^{(4,5)} \mathrm{Se}$ observa, además, que la cifra de niños nacidos con $\mathrm{VIH}$ ha disminuido significativamente en población urbana ${ }^{(6)}$, pero no necesariamente en contextos indígenas.

El estudio sobre las gestantes con $\mathrm{VIH}$ se concentra en la salud del nonato y la prevención de la transmisión vertical $^{(7)}$. Es necesario generar más información sobre el tema, especialmente en población indígena, conociendo como es que estas mujeres conciben a la enfermedad, qué prácticas de cuidado y prevención de la transmisión

\footnotetext{
Pontificia Universidad Católica del Perú. Lima, Perú.

Antropólogo

Recibido: : 01-10-14 Aprobado: 07-07-15
}

Citar como: Portocarrero J. VIH en gestantes indígenas: un reto para la salud pública peruana. Rev Peru Med Exp Salud Publica. 2015;32(3):546-54. 
vertical desarrollan, así como sus percepciones sobre el tratamiento antirretroviral de gran actividad (TARGA) y el papel de la medicina tradicional en la atención de su condición de salud. Esta revisión narrativa aborda la problemática de las gestantes indígenas con VIH haciendo un recuento de los principales hallazgos sobre el tema en el ámbito internacional, para luego revisar el estado de la investigación sobre el tema en el Perú. Para ello, se buscó entre literatura nacional e internacional sobre el tema de los últimos diez años a través de los buscadores PUBMED, JSTOR y la plataforma Research Gate, utilizando cinco términos de búsqueda en inglés, castellano y portugués: "VIH/SIDA" "población/ pueblo/ comunidades indígenas", "Amazonía", "gestantes indígenas" y "transmisión vertical". Se seleccionaron solo los artículos indexados. Se debe subrayar que el término "indígena" es problemático ya que se impone sobre una amplia y diversa variedad de grupos humanos con ciertos elementos en común, vinculados con los procesos de colonización europea, pero con diferencias tan marcadas que hacen discutible su comparabilidad. Es por ello que en esta revisión se indica el pueblo o región a la que pertenecen los hallazgos mencionados.

\section{HALLAZGOS EN EL ÁMBITO INTERNACIONAL: EFECTOS DEL VIH EN LA VIDA DE LAS GESTANTES}

El VIH tiene una serie de impactos negativos en la vida de las gestantes que sobrepasan los aspectos biomédicos. En el caso de las gestantes indígenas, los estudios encontrados registran dimensiones como el abandono de la pareja ${ }^{(8)}$, dificultades para el ejercicio de la maternidad, reducción de ingresos, ruptura de redes sociales, pérdida de estatus en la familia y comunidad ${ }^{(9)}$ así como estigma social ${ }^{(10)}$. La condición de $\mathrm{VIH}$ positivo puede afectar la identidad de la mujer y de la madre, no solo sobre cómo ella se autopercibe sino también sobre cómo es percibida e identificada por otros. Estos efectos pueden ser generados por la forma en que el entorno del paciente concibe a la enfermedad y la asociación con un comportamiento inmoral y el temor al contagio ${ }^{(11)}$. Existe una construcción social sobre el VIH que influye no solo en la forma cómo actúan estas mujeres sino también en la forma en que son percibidas y tratadas ${ }^{(12)}$. Se ha encontrado, además, que en muchos casos, la maternidad genera que las mujeres viviendo con VIH opten, o se vean obligadas, a revelar su situación de salud, con efectos tantos positivos como negativos en su entorno familiar y comunitario ${ }^{(13)}$.

Existe evidencia que demuestra que estos impactos trascienden a la gestante y afectan a su familia y futura descendencia. La presencia del virus del VIH no solo afecta a los pacientes sino a sus entornos familiares ${ }^{(14)}$, generando cambios en su organización, división de tareas y roles, acceso a recursos y posición en la comunidad. Del mismo modo, se ha encontrado la actitud hacia los recién nacidos hijos de madres con VIH puede ser variable e incluir situaciones como el rechazo y el temor ${ }^{(15)}$.

Dichos impactos negativos también afectan directamente las posibilidades de atención de su salud. Por ejemplo, estudios desarrollados en África subsahariana rural indican que muchas gestantes con VIH evitan acudir a los servicios de salud para no revelar su condición a la comunidad. Es, por tanto, importante mejorar las capacidades interculturales del personal de salud que las atiende para mejorar su adhesión así como la respuesta de la comunidad ${ }^{(16)}$.

\section{CUIDADO DE LAS MUJERES CON VIH}

Existe poca literatura sobre la maternidad y los cuidados en mujeres viviendo con $\mathrm{VIH}{ }^{(17)}$. Buena parte de las investigaciones sobre el tema se han concentrado en aspectos biomédicos relacionados principalmente sobre los efectos del VIH en el embarazo y el bienestar del feto o neonato. Se ha encontrado que la forma en que en que una mujer viviendo con VIH afronta su maternidad es definida tanto de manera personal y familiar como cultural e histórica ${ }^{(17)}$. La evidencia demuestra que es necesario entender no solo el perfil individual de estas mujeres, sino el contexto en el que viven. Esto incluye su familia, el acceso a información y los recursos en la comunidad, estrategias de subsistencia, entre otros aspectos ${ }^{(18,19)}$.

Se ha encontrado, además, que muchas mujeres indígenas en edad fértil o gestantes, viviendo con $\mathrm{VIH}$, manejan poca información respecto a cómo desarrollar una maternidad segura ${ }^{(20,21)}$. Buena parte de la atención que se da a una gestante o puérpera con VIH depende de consideraciones culturales que pueden poner en riesgo su salud y la de sus niños ${ }^{(22)}$. Las madres viviendo con $\mathrm{VIH}$, sus interpretaciones y prácticas en relación a la enfermedad, así como sus acciones de prevención, no solo dependen de la oferta de salud sino de sus entornos familiares y comunitarios, lo cual genera factores de riesgo y protección ${ }^{(23,24)}$. Parte de las acciones de cuidado y prevención desarrolladas durante el embarazo, parto o puerperio dependen de la información a la que ha tenido acceso la paciente y su familia. Esta puede ser parcial, fragmentada y diversa y provenir de diferentes fuentes, en un proceso complejo en el que se combinan diferentes discursos locales y foráneos ${ }^{(25)}$. El soporte familiar durante el embarazo y el cuidado del niño que ha nacido cumple un papel importante en el bienestar de gestantes y madres con esta enfermedad ${ }^{(26)}$. 
Las acciones de cuidado tienen que ver con acceso a recursos terapéuticos de diferentes sistemas de salud. Aunque la medicina tradicional y popular cumple una función importante en el tratamiento de los problemas de salud cotidianos de la población, poco se sabe de su uso en casos de VIH entre población indígena. Estudios desarrollados en África subsahariana han identificado la utilización de ciertas plantas medicinales y nutricionales en el manejo de infecciones oportunistas asociadas al VIH ${ }^{(27)}$, sin embargo, es necesario analizar la eficacia de estos recursos, su forma de preparación y sus métodos de administración.

En cuanto a la oferta de los servicios de salud públicos, se ha encontrado que buena parte de las necesidades de cuidado de las mujeres embarazadas y puérperas no son identificadas y atendidas por los servicios de salud y deben ser resueltos por ellas mismas y su entorno ${ }^{(22)}$. Muchas gestantes con VIH en poblaciones originarias no reciben, o reciben un limitado control médico de su situación de salud por diferentes motivos (28). Estudios demuestran que en muchos contextos en el que los varones no suelen acudir a los establecimientos de salud, tampoco acompañan a sus parejas en el tratamiento antirretroviral, ni están informados o involucrados en el tema ${ }^{(29)}$. La evidencia sugiere que, dado que las posibilidades de acceso a tratamiento y situación de las gestantes con $\mathrm{VIH}$ es un predictor importante de la condición de salud de sus futuros hijos, es importante desarrollar programas enfocados en prevenir la transmisión vertical y cuidar de la salud de los niños, hijos de madres VIH positivo ${ }^{(30)}$. Es necesario incrementar la educación y tratamiento culturalmente adecuado a gestantes ${ }^{(5,31)}$. El éxito relativo en el control de los índices de VIH/SIDA en población indígena australiana parece tener que ver con la inclusión de una estrategia de comunicación culturalmente apropiada, junto con la identificación temprana de los casos y la dotación de insumos y recursos para su atención.

\section{MUIERES INDÍGENAS CON VIH Y EXPECTATIVAS DE REPRODUCCIÓN}

La bibliografía existente sugiere que las mujeres viviendo con $\mathrm{VIH}$, sean indígenas o no, mantienen su deseo de ser madres y frecuentemente conciben niños después de ser diagnosticadas ${ }^{(32-35)}$. Pese a ello, existe poco conocimiento con relación a sus experiencias de vida, formas de interpretar la enfermedad, estrategias para cuidar y alimentar a sus niños y evitar la transmisión vertical (36). Se ha encontrado que la cultura influye en la decisión de procrear de mujeres viviendo con $\mathrm{VIH}^{37-}$ 39), ya que en muchos grupos sociales, especialmente en grupos indígenas, la maternidad forma parte de la identidad de género ${ }^{(40)}$. Se ha encontrado también que muchas mujeres viviendo con $\mathrm{VIH}$ optan por concebir debido a factores e influencia religiosa (41). Futuros estudios sobre el tema deben incluir factores culturales para mostrar una perspectiva holística del fenómeno.

\section{GESTANTES CON VIH Y TRANSMISIÓN VERTICAL}

Aunque ha sido poco estudiada en nuestro país, la transmisión vertical es una de las principales formas de adquisición del VIH en el Perú (42). Estudios internacionales consideran que las gestantes con $\mathrm{VIH}$ deben recibir tratamiento antirretroviral independientemente de su nivel de CD4, garantizando los estudios serológicos y la atención adecuada para prevenir la transmisión vertical ${ }^{(43)}$.

Se ha encontrado que en contextos de pobreza y sin acceso a información, las madres con VIH presentan una serie de incertidumbres sobre el cuidado de sus hijos frente a la exposición a la enfermedad y pueden desarrollar prácticas que generan riesgos de transmisión vertical ${ }^{(44)}$.

La lactancia materna es considerada, en la mayoría de grupos culturales, como un elemento fundamental de la experiencia de la maternidad y para el desarrollo del niño ${ }^{(45)}$. Las prácticas de alimentación de los infantes no solo tienen que ver con elecciones individuales, sino con normas sociales y culturales y de condiciones de vida que pueden evitar la postergación de la lactancia materna, que deben ser tomadas en cuenta en una estrategia que considere la reducción de transmisión vertical en población indígena ${ }^{(46,47)}$.

Se ha identificado que mujeres de pueblos indígenas y originarios que reciben tratamiento para el $\mathrm{VIH}$ no esperan a alcanzar niveles adecuados de CD4 para concebir y alimentar a sus hijos, dado que la maternidad y la lactancia son vistas como elementos esenciales para el desarrollo pleno de una mujer. Se ha encontrado, además, que muchas consideran que el tratamiento seguido es suficiente para poder concebir. Se observa al personal de salud como un obstáculo para la maternidad de las mujeres afectadas ${ }^{(48)}$.

En muchos casos, las tareas y responsabilidades de cuidado son transmitidas hacia abuelas debido a la muerte materna o la condición de salud de la madre (49). Muchas de las posibilidades de prevención de la transmisión vertical no dependen de la madre sino de su pareja y las relaciones que estos establecen. Se han encontrado, también, diferencias entre padres y madres sobre los sistemas de interpretación y actitud hacia el $\mathrm{VIH}$ y el riesgo de trasmisión a los recién nacidos ${ }^{(50)}$. 
La adecuación cultural de las estrategias de atención a gestantes indígenas australiana ha reducido la trasmisión vertical perinatal ${ }^{(51)}$.

\section{VIH EN POBLACIÓN INDÍGENA EN EL PERÚ}

En el Perú, el VIH es una epidemia concentrada que presenta los índices más elevados en la ciudad de Lima y centros urbanos portuarios de la Amazonía, particularmente entre hombres que tiene sexo con otros hombres ${ }^{(52-55)}$. Sin embargo, existen poblaciones, como las comunidades indígenas amazónicas, en donde esta cifra se está incrementando ${ }^{(56)}$.

Hay poca evidencia sobre la prevalencia de VIH y sus efectos en población indígena de Latinoamérica y el Perú (52,57-59). La información es diversa y se basa en estudios desarrollados con estrategias de análisis y muestreo diferentes, algunos de ellos con fuertes sesgos de selección de casos ocasionados al realizar mediciones en comunidades con brotes epidemiológicos que producen tasas más altas a la que resultarían si se seleccionaran otras comunidades dentro del mismo grupo étnico y territorio. Los primeros reportes de casos de VIH y SIDA en indígenas peruanos aparecieron en 2004, en población Chayahuita del Alto Amazonas, cuando la Dirección General de Epidemiología del MINSA recibió un reporte de la muerte de un paciente diagnosticado con SIDA perteneciente a una comunidad de este pueblo. En el año 2007, Zavaleta reportó una relativamente alta prevalencia de la enfermedad en una comunidad de este grupo ${ }^{(60)}$. En el 2008 Barletta ${ }^{(58)}$ identificó dos casos de VIH entre 282 personas, también entre Chayahuitas, encontró, además, que menos de la mitad de la población conocía el condón y solo un $20 \%$ reportaba haberlo usado alguna vez. Para el año 2012, Ormaeche ${ }^{(52)}$ registró una seroprevalencia bastante más baja que en los estudios anteriores, a partir de una investigación de mayor cobertura que incluía a gestantes y sus parejas de seis pueblos indígenas en cinco regiones del país. Pese a esta diversidad de resultados los estudios coinciden en señalar que la prevalencia de $\mathrm{VIH}$ en las comunidades indígenas amazónicas es mayor en los varones, y que la mayoría de casos de VIH identificados corresponden a varones que admitieron haber sostenido relaciones sexuales con otro hombre, por lo que la Dirección General de Epidemiología ${ }^{(61)}$ considera que el VIH entre la población indígena amazónica presenta una progresión que podría ser más rápida que en las áreas urbanas del país generando el riesgo de una epidemia.

La población indígena amazónica se encuentra dentro de los grupos poblacionales más afectados y vulnerables a la epidemia de $\mathrm{VIH}$, por ello deben ser consideradas de manera prioritaria en la formulación de las estrategias de prevención para los grupos clave ${ }^{(61)}$. La salud de la población indígena es altamente vulnerable debido a sus dificultades para acceder a servicios de salud; por una inadecuada oferta de salud y a la desarticulación de su sistema de salud tradicional, además de la pequeña escala y dispersión de su población ${ }^{(52)}$. El impacto del VIH en esta población podría ser mayor debido a su pequeño número poblacional; a su pobre conocimiento sobre la infección por el VIH y sobre su prevención; a las barreras económicas, culturales y lingüísticas que enfrentan para el acceso a los servicios así como para la aceptación de métodos de prevención conocidos y por el contacto cada vez más frecuente con las zonas urbanas, entre otros ${ }^{(61)}$. Se considera, además, que el VIH puede tener efectos devastadores en comunidades relativamente pequeñas ${ }^{(57)}$.

\section{FACTORES DE RIESGO IDENTIFICADOS}

Aunque la mayoría de investigaciones sobre $\mathrm{VIH}$ en población indígena en el Perú no pueden ser considerados estudios de riesgo en un sentido estricto, por ejemplo, no se utilizan casos controles ni se realizan estudios longitudinales, se indican una serie de factores que tienen que ver tanto con las características socioculturales de la población afectada y las debilidades de los servicios de salud disponibles así como por la presencia de nuevos actores y grupos poblaciones en sus territorios.

\section{FACTORES ASOCIADOS A LAS CARACTERISTICAS DE LA POBLACIÓN}

En cuanto a las características de la población, la bibliografía coincide en subrayar el papel del no uso de preservativos $(52,58,60)$ por diversas razones como el desconocimiento, la falta de disponibilidad en los establecimientos de salud y el rechazo por parte de la población ${ }^{(58,59)}$ Se mencionan también patrones de comportamiento sexual como la alta prevalencia de relaciones sexuales extramaritales ${ }^{(59)}$, el funcionamiento de la institución de la "entrega" ${ }^{(63)}$, una alta frecuencia autorreportada de varones que tienen sexo con otros varones ${ }^{(54,55,58,60)}$, el inicio sexual HSH ${ }^{(63)}$, una cultura sexual más "permisiva" ${ }^{(60)}$, la iniciación temprana de la actividad sexual, la institución matrimonial de la poliandria ${ }^{(60,63)}$, así como el rechazo y marginalización a personas homosexuales lo cual los obliga, en muchos casos, a abandonar sus comunidades para instalarse en zonas de mayor prevalencia desarrollando actividades que podrían ubicarlos en una situación de mayor exposición a la enfermedad ${ }^{(63)}$. Algunos de estos factores 
deben ser relativizados, particularmente los referidos a la dimensión sociocultural de la epidemiología de la enfermedad. Solo como ejemplo, se puede mencionar que la poliandria (un varón emparejado con más de una mujer, generalmente de generaciones diferentes, con quienes puede compartir una misma residencia) no puede ser entendida como parte de un comportamiento sexual promiscuo que algunos factores como "una cultura sexual más permisiva" no se encuentran bien definidos. Un segundo problema que considerar es el peso atribuido a los factores socioculturales frente a las condiciones de vida y reproducción de la población.

Un tercer elemento sumamente importante es el desconocimiento de la población con relación a la enfermedad. Se indica que en la población indígena amazónica existe poco conocimiento sobre la prevención, transmisión e infección del VIH y una baja aceptación de los métodos tradicionales utilizados para su prevención, por ejemplo, en un estudio realizado entre Chayahuitas, más de la mitad de los encuestados indicaba no saber de la existencia del VIH/SIDA y solo $15 \%$ de los que lo conocían indicaba que el uso de preservativos podía prevenir su transmisión ${ }^{(58,63)}$. Poco se ha investigado sobre el sistema de interpretación del VIH en población indígena. Es posible que existan diferentes discursos sobre la enfermedad que entremezclen explicaciones tradicionales sobre este padecimiento, con discursos religiosos y de medicina popular junto con interpretaciones biomédicas. El VIH es una enfermedad distante de la experiencia de buena parte de la población, quienes consideran que las enfermedades tienen manifestaciones somáticas evidentes. No obstante, se debe evitar el determinismo cultural de considerar que los sistemas de interpretación del VIH en estos pueblos definen exclusivamente sus comportamientos de riesgo y acciones de prevención y atención.

\section{CON RELACIÓN A LA OFERTA DE SALUD OFICIAL}

Por el lado de la oferta de salud, la población indígena adolece de una serie de problemas como la actitud del personal de salud para interactuar con miembros de una cultura diferente a la suya, que el Seguro Integral de Salud (SIS) no favorezca aún a los pueblos indígenas y campesinos más alejados, así como el desabastecimiento de medicamentos, insumos y equipamiento para brindarles una adecuada atención ${ }^{(64)}$.

A nivel central, debido a su escasa densidad poblacional, el Ministerio de Salud (MINSA) no prioriza la atención de salud de las poblaciones indígenas que se encuentran en las zonas más alejadas de las grandes ciudades asignando limitados recursos a sus establecimientos de salud. Asimismo, las estrategias sanitarias diseñadas a nivel central no se adaptan a las estrategias de vida, cultura y necesidades sentidas por la población indígena.

En cuanto al control del VIH/SIDA en población indígena, se ha indicado que existen dificultades para proveer de información adecuada a esta población ${ }^{(57,62)}$, realizar pruebas rápidas y confirmatorias, problemas para la atención de los casos detectados ${ }^{(63)}$, migración de retorno de pacientes con $\mathrm{VIH}^{\left({ }^{63}\right)}$ y la no adaptación de las estrategias de prevención y control del $\mathrm{VIH}$ a las características socioculturales y condiciones de vida de la población indígena amazónica ${ }^{(63)}$.

\section{FACTORES DE CONTEXTO}

Muchos estudios no consideran el contexto y los factores estructurales que están detrás de este problema $(63,65)$. Estos operan a diferentes niveles (individual, interpersonal, comunal) delineando condiciones de vida que incrementan la trasmisión del $\mathrm{VIH}$ :

- Riesgo generado a partir de contacto, directo e indirecto, de indígenas con ciudades grandes e intermedias ${ }^{(58)}$.

- Migración constante de población pobre de costa y andes, industrias extractivas ${ }^{(65)}$. Crecimiento de ciudades intermedias con población móvil (63).

- Población móvil que establece fluidos y diferentes, pero desiguales intercambios con otros grupos poblaciones. Se ha encontrado que varones indígenas que tienen sexo con otros hombres y dejan sus comunidades para migrar hacia ciudades a lo largo del río Amazonas establecen comportamientos de riesgo para contraer $\mathrm{VIH}$, como el sexo sin protección con MSM mestizos (Alva y Orellana, 2012) debido a situaciones de discriminación y débiles redes sociales de soporte ${ }^{(65)}$.

- Discriminación y relaciones de subordinación hacia población indígena amazónica ${ }^{(65,66)}$.

Se evidencia la necesidad de desarrollar un modelo de análisis basado en la identificación de variables externas y externas a las comunidades, de carácter histórico, que considere y articule factores sociales, culturales, políticos, económicos y ecológicos en sus niveles macro, meso y micro.

\section{ITINERARIOS TERAPÉUTICOS Y VIH EN POBLACIÓN INDÍGENA AMAZÓNICA}

Poco se conoce sobre los itinerarios y recursos terapéuticos de la población afectada. Un estudio realizado por UNFPA e IES en el año 2009 encuentra que los itinerarios 
terapéuticos de indígenas shipibo conibo y yine se concentraba en la medicina tradicional y doméstica. Se encontró, además, que los itinerarios terapéuticos eran sumamente restringidos a aliviar algunos síntomas, gran desinformación y un escenario de complejidad discursiva en el que la población tiene un alto nivel de incertidumbre entre discursos diversos respecto a la causalidad y posibilidades de atención del VIH. Además, en este estudio se identificaron terapeutas tradicionales que afirmaban poder tratar y curar la enfermedad con plantas medicinales ${ }^{(59)}$. Desde nuestra experiencia, este tipo de ofertas puede complementar, pero también competir con la del TARGA, generando problemas para la adhesión al tratamiento provisto por el Estado.

\section{GESTANTES INDÍGENAS EN EL PERÚ Y EL VIH}

Aunque la investigación en el tema nos ayudaría a entender mejor los procesos de transmisión sexual y vertical del $\mathrm{VIH}$, en nuestro país solo existe un estudio sobre gestantes indígenas y sus parejas ${ }^{(52)}$. En este se encontró que buena parte de estas gestantes son bastante jóvenes (un promedio de 14,9 años) y menos del $7 \%$ reconocía que el contacto sexual con extraños podía ser un factor de riesgo de transmisión de las ITS. Además, solo un $22,8 \%$ de ellas indicó al preservativo como un medio para prevenir este tipo de enfermedad ${ }^{(52)}$.

\section{CONCLUSIONES}

De acuerdo con representantes de la Estrategia Sanitaria Nacional de Prevención y Control de las ITS, VIH/SIDA y de la Dirección General de Epidemiología, es posible que exista un subregistro en las cifras oficiales de VIH en población indígena. Los datos manejados por ambas instituciones son agregados a nivel regional y no necesariamente coinciden entre sí, ni con los registrados por las DIRESA. Tampoco se cuenta con un registro de pertenencia étnica de las personas diagnosticadas. Por el lado de los pacientes, muchos de ellos abandonan los tratamientos y migran fuera de sus localidades evitando el estigma que acompaña a esta enfermedad.

El balance antes expuesto nos permite identificar una serie de vacíos y necesidades de investigación y acción:

- Estudios de seroprevalencia y riesgo en mujeres indígenas en edad reproductiva de mayor escala y representatividad

- Investigación sobre sistemas de interpretación del VIH en mujeres indígenas y población indígena en general

- Análisis de itinerarios terapéuticos y recursos locales para la atención del VIH en población indígena

- Estudios sobre la adecuación sociocultural y logísticas de la estrategia de control y prevención del VIH en población indígena

- Investigación sobre los efectos del VIH sobre grupos de riesgos como gestantes, ancianos y neonatos.

Este tipo de estudios permitirá mejorar la respuesta de salud pública frente al problema de gestantes con $\mathrm{VIH}$ en remotas comunidades nativas amazónicas con intervenciones que se adecúen a la cultural y modo de vida indígena, identificando los alcances y límites de las estrategias utilizadas por el Estado para controlar y atender los casos, evaluando sus problemas ${ }^{(58-60,62)}$. En estas estrategias se deben tomar en cuenta cuestiones de género, ya que una buena proporción de mujeres en muchos grupos indígenas no maneja el castellano y tiene una reducida capacidad de decisión fuera del ámbito doméstico ${ }^{(58)}$. Se evidencia la necesidad de un urgente enfoque médico antropológico para detener la transmisión de $\mathrm{VIH}$ en las comunidades indígenas de la amazonia (62).

Fuentes de financiamiento: Centro Nacional de Salud Intercultural, Instituto Nacional de Salud.

Conflictos de interés: el autor declara no tener conflictos de interés.

\section{REFERENCIAS BIBLIOGRÁFICAS}

1. Milliez J. Rights to safe motherhood and newborn health: ethical issues. Int J Gynaecol Obstet. 2009 Aug;106(2):110-1. doi: 10.1016/j. ijgo.2009.03.019.

2. Garenne M, McCaa R, Nacro K. Maternal mortality in South Africa in 2001: From demographic census to epidemiological investigation. Popul Health Metr. 2008;6(4):1-13.

3. Moodley J, Pattinson RC, Baxter C, Sibeko S, Abdool Karim Q.
Strengthening HIV services for pregnant women: an opportunity to reduce maternal mortality rates in Southern Africa/sub-Saharan Africa. BJOG. 2011;118(2):219-25. doi: 10.1111/j.1471-0528.2010.02726.x.

4. McIntyre J. Mothers infected with HIV. Reducing maternal death and disability during pregnancy. Br Med Bull. 2003;67(1):127-35.

5. Habib NA, Daltveit AK, Bergsjø P, Shao J, Oneko O, Lie RT. Maternal
HIV status and pregnancy outcomes in northeastern Tanzania: a registry-based study. BJOG. 2008;115(5):616-24. doi: 10.1111/j.1471-0528.2008.01672.x.

6. Velázquez C. Resultados de la aplicación de tres guías nacionales para prevenir la transmisión vertical del VIH en el Instituto Nacional Materno Perinatal. Lima, Perú. Rev Peru Med Exp Salud Publica. 2011;28(3):492-6.

7. Loutfy MR, Sonnenberg-Schwan U, Margolese S, Sherr L; Women 
for Positive Action. A review of reproductive health research, guidelines and related gaps for women living with HIV. AIDS Care. 2013;25(6):657-66. doi: 10.1080/09540121.2012.733332.

8. Moyo ON, Kawewe SM. Lone motherhood in Zimbabwe: the socioeconomic conditions of lone parents and their children. Soc Work Public Health. 2009;24(1-2):161-77. doi: $10.1080 / 19371910802569732$.

9. Wilson S. 'When you have children, you're obliged to live': motherhood, chronic illness and biographical disruption. Sociol Health Illn. 2007;29(4):610-26.

10. Cook RJ, Dickens BM. Reducing stigma in reproductive health. Int J Gynaecol Obstet. 2014;125(1):89-92. doi: 10.1016/j.ijgo.2014.01.002.

11. Simon A, Laprevotte M, Edeb N. [The multiple vulnerabilities of women with AIDS]. Rev Infirm. 2013;(190):25-7. [Articulo en frances]

12. Naidu T Sliep Y, Dageid W. The social construction of identity in HIV/ AIDS home-based care volunteers in rural KwaZulu-Natal, South Africa. SAHARA J. 2012;9(2):113-26. doi: 10.1080/17290376.2012.683585.

13. Iwelunmor J, Zungu N, Airhihenbuwa CO. Rethinking HIV/AID disclosure among women within the context of motherhood in South Africa. Am J Public Health. 2010;100(8):1393-9. doi: 10.2105/AJPH.2009.168989.

14. Harrison A, Short SE, Tuoane-Nkhasi M. Re-focusing the gender lens: caregiving women, family roles and HIV/AIDS vulnerability in Lesotho. AIDS Behav. 2014;18(3):595-604. doi: 10.1007/s10461-013-0515-z.

15. Oosterhoff P, Anh NT, Yen PN, Wright P, Hardon A. HIV-positive mothers in Viet Nam: using their status to build support groups and access essential services. Reprod Health Matters. 2008;16(32):162-70. doi: 10.1016/ S0968-8080(08)32408-2.

16. Turan JM, Miller S, Bukusi EA, Sande J, Cohen CR. HIV/AIDS and maternity care in Kenya: how fears of stigma and discrimination affect uptake and provision of labor and delivery services. AIDS Care. 2008;20(8):938-45. doi: $10.1080 / 09540120701767224$.

17. Kennedy VL, Serghides L, Raboud JM, Su D, Blitz S, Hart TA, et al. The importance of motherhood in
HIV-positive women of reproductive age in Ontario, Canada. AIDS Care. 2014;26(6):777-84. doi: 10.1080/09540121.2013.855295.

18. Vidal L, Loû AD. [AIDS and social situations of women in Africa: from notions to research methods]. Sante. 2001;11(4):265-72.

19. Anderson J. Women and HIV: motherhood and more. Curr Opin Infect Dis. 2012;25(1):58-65. doi: 10.1097/QCO.0b013e32834ef514.

20. Mushi DL, Mpembeni RM, Jahn A. Knowledge about safe motherhood and HIV/AIDS among school pupils in a rural area in Tanzania. BMC Pregnancy Childbirth. 2007;7:5.

21. De Moura EL, Praça Nde S. [HIV perinatal transmission: expectations and actions of HIV-positive pregnant women]. Rev Lat Am Enfermagem. 2006;14(3):405-13.

22. Sofolahan YA, Airhihenbuwa CO. Cultural expectations and reproductive desires: experiences of South African women living with HIV/AIDS (WLHA). Health Care Women Int. 2013;34(3-4):263-80. doi: $10.1080 / 07399332.2012 .721415$.

23. Vescovi G, Pereira M, Levandowski DC. Protective factors in the experience of pregnancy and motherhood among Brazilian adolescents living with HIV: A case-series report. J Assoc Nurses AIDS Care. 2014;25(6):541-54. doi: 10.1016/j.jana.2014.02.005.

24. Treisman K, Jones FW, Shaw E. The experiences and coping strategies of United Kingdom-based African women following an HIV diagnosis during pregnancy. J Assoc Nurses AIDS Care. 2014;25(2):145-57. doi: 10.1016/j.jana.2013.01.008

25. Moses S, Tomlinson M. The fluidity of disclosure: a longitudinal exploration of women's experience and understanding of HIV disclosure in the context of pregnancy and early motherhood. AIDS Care. 2013;25(6):667-75. doi: $10.1080 / 09540121.2012 .736606$.

26. Vescovi G, Pereira M, Levandowski DC. Protective factors in the experience of pregnancy and motherhood among Brazilian adolescents living with HIV: A case-series report. J Assoc Nurses AIDS Care. 2014;25(6):541-54. doi: 10.1016/j.jana.2014.02.005.

27. Mugisha MK, Asiimwe S, Namutebi A, Borg-Karlson AK, Kakudidi EK.
Ethnobotanical study of indigenous knowledge on medicinal and nutritious plants used to manage opportunistic infections associated with HIV/AIDS in western Uganda. J Ethnopharmacol. 2014;155(1):194-202. doi: 10.1016/j. jep.2014.05.012.

28. Delva W, Yard E, Luchters S, Chersich MF, Muigai E, Oyier V, et al. A Safe Motherhood project in Kenya: assessment of antenatal attendance, service provision and implications for PMTCT. Trop Med Int Health. 2010;15(5):584-91. doi: 10.1111/j.1365-3156.2010.02499.x.

29. Adeleye OA, Chiwuzie J. "He does his own and walks away" perceptions about male attitudes and practices regarding safe motherhood in Ekiadolor, Southern Nigeria. Afr J Reprod Health. 2007;11(1):76-89.

30. Nakiyingi JS, Bracher M, Whitworth JA, Ruberantwari A, Busingye J, Mbulaiteye SM, et al. Child survival in relation to mother's HIV infection and survival: evidence from a Ugandan cohort study. AIDS. 2003;17(12):1827-34.

31. Ward J, Costello-Czok M, Willis J, Saunders M, Shannon C. So far, so good: Maintenance of prevention is required to stem HIV incidence in Aboriginal and Torres Strait Islander communities in Australia. AIDS Educ Prev. 2014;26(3):267-79. doi: 10.1521/aeap.2014.26.3.267.

32. Kanniappan S, Jeyapaul MJ, Kalyanwala S. Desire for motherhood: exploring HIV-positive women's desires, intentions and decisionmaking in attaining motherhood. AIDS Care. 2008;20(6):625-30. doi: $10.1080 / 09540120701660361$.

33. Nóbrega AA, Oliveira FA, Galvão MT, Mota RS, Barbosa RM, Dourado I, et al. Desire for a child among women living with HIV/AIDS in northeast Brazil. AIDS Patient Care STDS. 2007;21(4):261-7.

34. Santos NJ, Buchalla CM, Fillipe EV, Bugamelli L, Garcia S, Paiva V. [Reproduction and sexuality in HIVpositive women, Brazil]. Rev Saude Publica. 2002;36(4 Suppl):12-23. [Articulo en portugués]

35. Kennedy VL, Serghides L, Raboud JM, Su D, Blitz S, Hart TA, et al. The importance of motherhood in HIV-positive women of reproductive 
age in Ontario, Canada. AIDS Care. 2014;26(6):777-84. doi: $10.1080 / 09540121.2013 .855295$.

36. Liamputtong P, Haritavorn N. My life as Mae Tid Chua [mothers who contracted HIV disease]: Motherhood and women living with HIV/AIDS in central Thailand. Midwifery. 2014;30(12):1166-72. doi: 10.1016/j. midw.2014.04.003.

37. Nattabi B, Li J, Thompson SC, Orach CG, Earnest J. A systematic review of factors influencing fertility desires and intentions among people living with HIV/AIDS: implications for policy and service delivery. AIDS Behav. 2009 Oct;13(5):949-68. doi: 10.1007/ s10461-009-9537-y.

38. Barnes DB, Murphy S. Reproductive decisions for women with HIV: motherhood's role in envisioning a future. Qual Health Res. 2009;19(4):481-91. doi: $10.1177 / 1049732309332835$.

39. Cooper D, Harries J, Myer L, Orner $\mathrm{P}$, Bracken $\mathrm{H}$, Zweigenthal V. "Life is still going on": reproductive intentions among HIV-positive women and men in South Africa. Soc Sci Med. 2007;65(2):274-83.

40. De Carvalho FT, Piccinini CA. [Historical aspects, maternity and HIV infection in women]. Cien Saude Colet. 2008;13(6):1889-98. [Articulo en portugués]

41. De La Cruz NG, Davies SL, Stewart KE. Religion, relationships and reproduction: correlates of desire for a child among mothers living with HIV. AIDS Behav. 2011;15(6):1233-42. doi: 10.1007/s10461-010-9788-7.

42. Alarcón Villaverde J, Romaní Romaní F, Montano Torres S, Zunt JR. Transmisión vertical de HTLV-1 en el Perú. Rev Peru Med Exp Salud Publica. 2011;28(1):101-8.

43. Expert Panel Secretariat of the National AIDS Plan (PNS); Spanish Society of Gynecology and Obstetrics (SEGO); AIDS Study Group (GeSIDA); Spanish Society of Pediatric Infectious Diseases (SEIP), Polo Rodríguez R, Muñoz Galligo E, et al. Executive summary of the Consensus Statement on monitoring HIV: pregnancy, birth, and prevention of mother-tochild transmission. Enferm Infecc Microbiol Clin. 2014;32(5):311-9. doi: 10.1016/j.eimc.2013.12.006.
44. Galvão MT, da Cunha GH, Machado MM. [Dilemmas and conflitcs of being a mother with HIV/AIDS]. Rev Bras Enferm. 2010;63(3):371-6. [Articulo en portugués]

45. Hebling EM, Hardy E. Feelings related to motherhood among women living with HIV in Brazil: a qualitative study. AIDS Care. 2007;19(9):1095-100.

46. Chinkonde JR, Hem MH, Sundby J. HIV and infant feeding in Malawi: public health simplicity in complex social and cultural contexts. BMC Public Health. 2012;12:700. doi: 10.1186/1471-2458-12-700.

47. Reed E, Silverman JG, Stein B, Erausquin JT, Biradavolu M, Rosenberg A, et al. Motherhood and HIV risk among female sex workers in Andhra Pradesh, India: the need to consider women's life contexts. AIDS Behav. 2013;17(2):543-50. doi: 10.1007/ s10461-012-0249-3.

48. Awiti Ujiji O, Ekström AM, Ilako F, Indalo D, Rubenson B. "I will not let my HIV status stand in the way." Decisions on motherhood among women on ART in a slum in Kenya- a qualitative study. BMC Womens Health. 2010;10:13. doi: 10.1186/1472-6874-10-13.

49. Hughes CB, Caliandro G. Empowerment: a case study of a grandmother caring for her HIVpositive grandchild. J Assoc Nurses AIDS Care. 2000;11(5):29-38.

50. Tijou Traoré A, Querre M, Brou $H$, Leroy V, Desclaux A, Desgrées-du-Loû A. Couples, PMTCT programs and infant feeding decision-making in Ivory Coast. Soc Sci Med. 2009;69(6):830-7. doi: 10.1016/j.socscimed.2009.06.001.

51. Gilles MT, Dickinson JE, Cain A, Turner KA, McGuckin R, Loh R. et al. Perinatal HIV transmission and pregnancy outcomes in indigenous women in Western Australia. Aust N Z J Obstet Gynaecol. 2007;47(5):362-7.

52. Ormaeche M, Whittembury A, Pun M, Suarez-Ognio L. Hepatitis B virus, syphilis, and HIV seroprevalence in pregnant women and their male partners from six indigenous populations of the Peruvian Amazon Basin, 2007-2008. Int J Infect Dis. 2012;16(10):e724-30. doi: 10.1016/j. ijid.2012.05.1032.

53. Beyrer C, Baral SD, Walker D, Wirtz AL, Johns B, Sifakis F. The expanding epidemics of HIV type 1 among men who have sex with men in low- and middle-income countries: diversity and consistency. Epidemiol Rev. 2010;32:137-51. doi: 10.1093/epirev/ mxq011.

54. Cáceres CF, Mendoza W. The national response to the HIV/AIDS epidemic in Peru: accomplishments and gaps-- a review. J Acquir Immune Defic Syndr. 2009;51 Suppl 1:S60-6. doi: 10.1097/ QAI.0b013e3181a66208.

55. Cárcamo CP, Campos PE, García PJ, Hughes JP, Garnett GP, Holmes KK. Prevalences of sexually transmitted infections in young adults and female sex workers in Peru: a national population-based survey. Lancet Infect Dis. 2012;12(10):765-73. doi: 10.1016/S1473-3099(12)70144-5.

56. García PJ, Bayer A, Cárcamo CP. The changing face of HIV in Latin America and the Caribbean. Curr HIV/AIDS Rep. 2014;11(2):146-57.doi: 10.1007/ s11904-014-0204-1.

57. Zavaleta C, Mujica J, Ypanaqué PJ, Cueva N. Infecciones de transmisión sexual y VIH/SIDA en comunidades nativas de la Amazonía peruana: consideraciones culturales. Rev Peru Med Exp Salud Publica. 2007;24(3):315-6.

58. Bartlett EC, Zavaleta C, Fernández C, Razuri H, Vilcarromero S, Vermund $\mathrm{SH}$, et al. Expansion of HIV and syphilis into the Peruvian Amazon: a survey of four communities of an indigenous Amazonian ethnic group. Int J Infect Dis. 2008;12(6):e89-94. doi: 10.1016/j.ijid.2008.03.036.

59. Perú, Ministerio de Salud; Fondo de Población de las Naciones Unidas. Una aproximación cualitativa a la prevención del VIH-Sida en dos comunidades nativas de Ucayali. Lima: Instituto de Educación y Salud; 2009.

60. Zavaleta C, Fernández C, Konda K, Valderrama Y, Vermund SH, Gotuzzo E. High prevalence of HIV and syphilis in a remote native community of the Peruvian Amazon. Am J Trop Med Hyg. 2007;76(4):703-5.

61. Perú, Ministerio de Salud; Dirección General de Epidemiología. Análisis de la situación epidemiológica del VIH/ SIDA en el Perú - 2013. Lima: JB GRAFIC; 2013.

62. Grández-Urbina JA, Fernández-Luque JL. Etnias de la Amazonía en riesgo de enfermedades de transmisión sexual, 
una realidad en nuestro país. Rev Med Hered. 2012;23(1):70-6.

63. Orellana ER, Alva IE, Cárcamo CP, García PJ. Structural factors that increase HIV/STI vulnerability among indigenous people in the Peruvian amazon. Qual Health Res. 2013;23(9):1240-50. doi: $10.1177 / 1049732313502129$.

64. Perú, Ministerio de Salud; Instituto Nacional de Salud; Centro Nacional de Salud Intercultural. Plan General de la Estrategia Sanitaria Nacional. Salud de los Pueblos Indígenas 2009 - 2012. Lima: MINSA; 2009.

65. Alva I, Orellana R. Social and contextual factors that influence HIV risk behaviors among indigenous MSM in the Peruvian Amazon. Retrovirology. 2012;9(Suppl 1):112.

66. Alva I. Sex on Amazonian riverboats: A neglected public health problem.
University of Washington. Seattle, Estados Unidos; 2013.

Correspondencia: Julio Portocarrero Gutiérrez Dirección: Pontificia Universidad Católica del Perú. Departamento de Ciencias Sociales. Av. Universitaria 1801, Lima 32. Teléfono (511) 6262000

Correo electrónico: juliocesarportocarrero@ hotmail.com

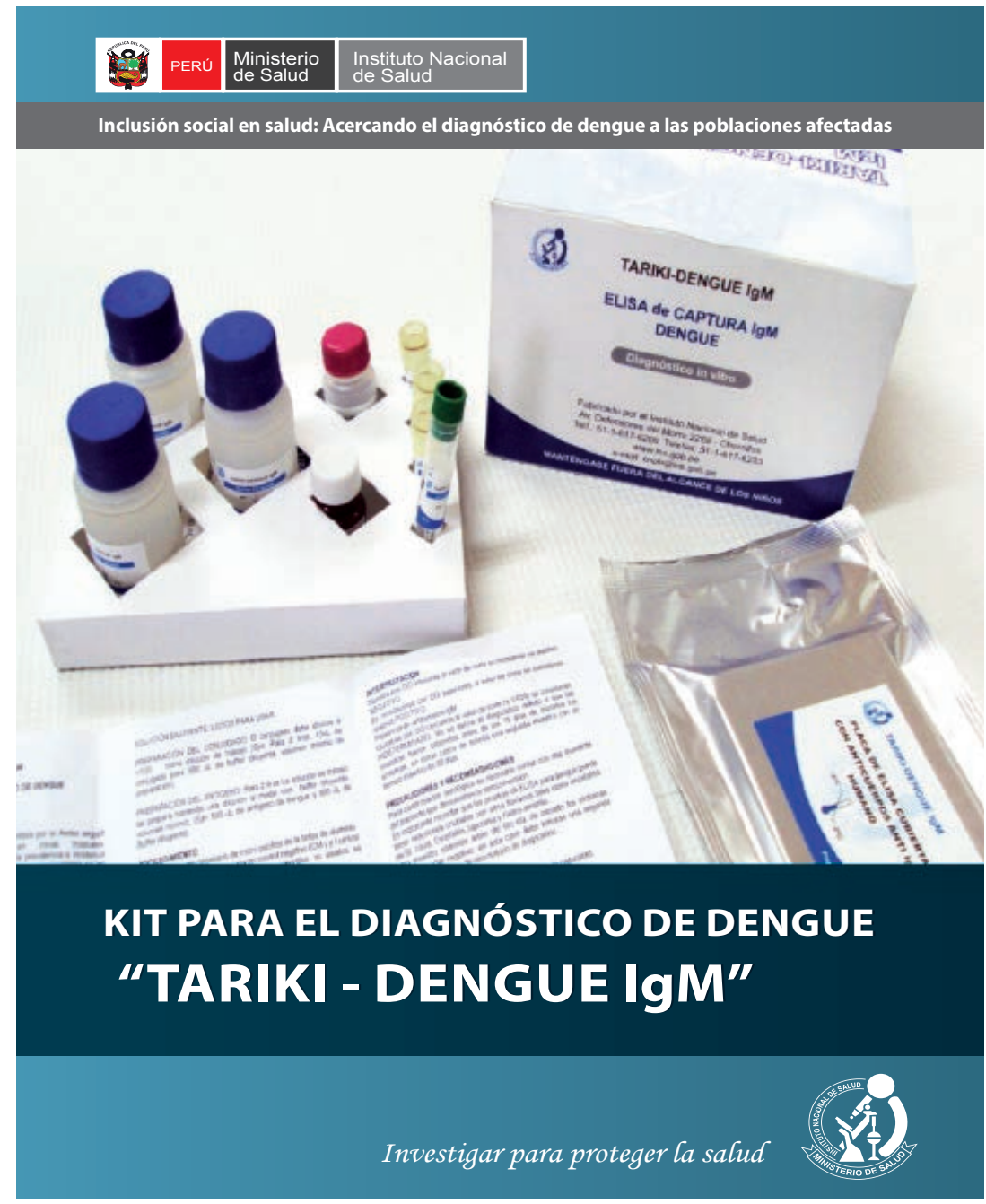

